\title{
Dietary supplement use among cancer survivors and the general population: a nation-wide cross-sectional study
}

\author{
Sihan Song ${ }^{1}$, Jiyoung Youn ${ }^{1}$, Yun Jung Lee ${ }^{1}$, Minji Kang ${ }^{1}$, Taisun Hyun ${ }^{2}$, YoonJu Song ${ }^{3}$ and Jung Eun Lee ${ }^{1 *}$ (D)
}

\begin{abstract}
Background: Use of dietary supplements among cancer survivors is common and controversial, but information on the amount of nutrients from supplements among cancer survivors is limited. We examined the amount of nutrients and their contribution to total nutrient intake from supplements and compared these data between cancer survivors and cancer-free individuals. We also identified factors associated with supplement use among cancer survivors.
\end{abstract}

Methods: We identified 400 cancer survivors and 10,387 cancer-free individuals, aged $\geq 19$ years, from the fifth Korea National Health and Nutrition Examination Survey (KNHANES) V-1, 2 (2010, 2011). We calculated the amount of nutrients consumed from foods and supplements, the percent contributions of supplement nutrients to total nutrient intakes and cancer survivors' nutrient intakes relative to the Estimated Average Requirements (EARs) and the Tolerable Upper Intake Levels (ULs). We examined factors associated with supplement use among cancer survivors.

Results: We found that $33.3 \%$ of cancer survivors and $22.1 \%$ of cancer-free individuals reported the use of dietary supplements. Compared to cancer-free individuals, cancer survivors had higher intakes of riboflavin, folate, and iron from foods ( $p<0.05$ for each), and higher intakes of calcium $(p=0.05)$ and vitamin $C(p=0.01)$ from foods and supplements.

The similar pattern was observed for the percent contributions to total nutrient intake. Cancer survivors had higher proportion of participants below EARs than cancer-free individuals for thiamin and niacin ( $p<0.05$ for each). The proportions of cancer survivors below the EARs were $61.2 \%$ for calcium, $49.1 \%$ for riboflavin, and $43.5 \%$ for folate and the proportions of cancer survivors above the ULs were 3.3\% for iron, and 2.3\% for vitamin A. For female cancer survivors, education above an elementary school level, moderate physical activity, low vegetable intake, and high circulating vitamin D levels were associated with supplement use. For male cancer survivors, living in an urban area, no consumption of alcohol, and lower energy intake, were associated with supplement use.

Conclusions: Korean cancer survivors have higher rate of dietary supplement use and higher contribution from supplements to total nutrient intake than cancer-free individuals. Demographic and lifestyle factors were associated with supplement use among cancer survivors.

Keywords: Cancer survivors, Dietary supplement use, Korea National Health and Nutrition Examination Survey

\section{Background}

Cancer survivors tend to start taking dietary supplements after receiving a diagnosis of cancer [1-4]. Previous studies have reported that, among cancer survivors, a high desire for personal control was associated with taking new dietary supplements [1] and the most commonly reported

\footnotetext{
* Correspondence: jungelee@snu.ac.kr

'Department of Food and Nutrition, Seoul National University, Gwanak-ro 1, Gwanak-gu, Seoul 08826, Republic of Korea

Full list of author information is available at the end of the article
}

reasons for dietary supplement use were to improve health (e.g., immune system) and prevent disease [3, 4]. A previous systematic review reported a tendency for a higher prevalence of vitamin and mineral supplement use among cancer survivors (64-81\%) than general US adults $(52 \%)$ [5]. Furthermore, according to this review, the prevalence of dietary supplement use was relatively higher in breast cancer survivors than other cancer sites, and high education levels and female sex were associated with dietary supplement use among cancer survivors [5]. 
Dietary supplement use in cancer care remains controversial [6-8]. Cancer patients who lost significant weight before radiotherapy or chemotherapy treatment are often offered nutritional supplements along with enteral tube feeding [9], whereas there are concerns for herb-drug interactions and potential detrimental effects of antioxidants on cancer treatment [6-8]. The American Cancer Society guidelines suggested that cancer survivors should assess their nutrient deficiency first, and avoid to take excessive amount of nutrients from dietary supplements [7]. Given limited research on the amount of nutrients consumed from dietary supplements among cancer survivors, it is important to examine whether their nutrient levels are adequate.

However, only a few survey studies have reported the amount of nutrients or the contribution of dietary supplements to total nutrient intakes among cancer survivors. A French study calculated nutrient intakes from foods and supplements among 270 cancer survivors, and found that the contribution of vitamin or mineral supplement to total nutrient intakes ranged from $0 \%$ for sodium and potassium to $79 \%$ for vitamin D [3]. A US study of 753 cancer survivors reported that the proportion consuming amounts below Estimated Average Requirements (EARs) among 559 supplement users was substantially reduced after the addition of nutrient intakes from dietary supplements: vitamin E (81\% to $12 \%)$, magnesium (77\% to $40 \%$ ), vitamin C (47\% to $9 \%$ ), and vitamin A (45\% to $8 \%$ ) [10]. In that study, the proportion consuming amounts above Tolerable Upper Intake Levels (ULs) was less than 10\% [10]. Another US study of 435 breast cancer survivors also found that less than $5 \%$ of 352 dietary supplement users consumed nutrient intakes exceeding ULs [11].

Cancer is the leading cause of death in South Korea [12]. The estimated cumulative risk of developing cancer during a lifetime is $36.2 \%$, and thyroid cancer is the most commonly diagnosed cancer, followed by stomach, colorectal, lung, and breast cancer, as reported in 2014 [13]. Dietary supplement use is also common among Korean adults. The age-standardized prevalence of dietary supplement use for at least 2 weeks during the past year among Korean adults increased from 25.7\% in 2005 to $41.5 \%$ in 2015 [14]. Several studies found that dietary supplements were commonly used among Korean cancer patients: the proportion of dietary supplement use was $53.2 \%$ and $78.1 \%$ among 126 and 105 gastrointestinal cancer patients, respectively $[15,16]$, and the proportion of vitamin/mineral use was $24.1 \%$ and $27.1 \%$ among 339 and 288 breast cancer patients, respectively $[17,18]$. However, to our knowledge, the amount of nutrients consumed and the nutritional contribution of dietary supplement to total nutrient intakes have not been reported among Korean cancer survivors. No information on the amount or contribution of nutrients consumed from dietary supplements among Korean cancer survivors may preclude researchers and health professionals from considering further investigation or intervention on cancer survivor's supplement use.

Therefore, we aimed to examine dietary supplement intake and its contribution to nutrient intake among cancer survivors using a database of dietary supplements, which is part of the fifth Korea National Health and Nutrition Examination Survey (KNHANES V-1,2), 2010 and 2011 , nationwide database $[19,20]$. We calculated the nutritional contribution of dietary supplements among the general population to compare with cancer survivors. Furthermore, we identified the characteristics of dietary supplement users among Korean cancer survivors.

\section{Methods}

Data source and study population

We included participants of the KNHANES V-1,2 (2010, 2011), a population-based cross-sectional nation-wide survey [20]. The KNHANES is conducted annually by the Korea Centers for Disease Control and Prevention (KCDC) to assess the health and nutritional status of Koreans. Sampling of non-institutionalized Korean citizens occurs via a multistage clustered probability design, and the health and nutritional information of participants is gained from health interviews, clinical examinations, and nutrition surveys. A detailed description of the study is provided elsewhere [21]. The health interviews and examinations were conducted in the mobile examination center (MEC). Information on smoking, alcohol use, and physical activity was collected via a selfadministered questionnaire. Housing characteristics and details of medical conditions and socioeconomic status were obtained by face-to-face interviews. Body mass index (BMI, $\mathrm{kg} / \mathrm{m}^{2}$ ) was calculated using weight and height, which were measured during the health examination. Fasting blood serum and urine samples were collected from participants aged $\geq 10$ years to obtain biochemical profiles. Dietary supplement use and dietary intake were obtained by face-to-face interviews conducted in the homes of the participants [21]. We used data from one-day 24-h dietary recalls to estimate nutrient intake from foods and dietary supplements. Energy and nutrient intakes from foods were calculated using the Korean National Rural Living Science Institute (KNRLSI) and Korea Health Industry Development Institute (KHIDI) databases [20, 22]. We estimated folate intake from 24-h dietary recalls using the folate database developed by Kim JH et al. [23] and Yon M et al. [24]. Non-quantitative food frequency questionnaires (FFQs) were used to estimate the daily servings of vegetables, fruits, and red and processed meats.

Of 17,476 participants identified from the KNHANES V-1, 2 (2010-2011) survey, we excluded those with 1) 
age $<19$ years, 2) no information on 24-h dietary recalls, 3) no information from a physician regarding cancer diagnosis, or 4) implausible energy intake (above the $\log$-transformed mean $\pm 3 \mathrm{SD}$ ). As a result, a total of 10,787 adults aged $\geq 19$ years were eligible for the study. All participants signed an informed consent form. Ethics approval was obtained from the KCDC Institutional Review Board (IRB) Ethics Committee (2010-02CON-21-C, 2011-02CON-06-C) [20].

\section{Ascertainment of cancer diagnosis}

Trained interviewers asked the participants, during faceto-face health interviews, whether they had ever been diagnosed with cancer by a physician. The participants reporting a history of cancer were asked for the site of cancer and the age when cancer was diagnosed [21]. We calculated survival time since the diagnosis of cancer by subtracting the age at the first diagnosis of cancer from the current age.

\section{Calculation of the nutrient amounts from dietary supplements}

We defined dietary supplement use as the use of any dietary supplement on the day preceding the date of the survey. The participants were otherwise categorized as non-users. Trained interviewers asked the participants about their dietary supplement use, including type, product name, manufacturing company, distributor, frequency, and amount, when they administered the 24-h dietary recalls during the face-to-face interview [19]. Using the dietary supplement database, we calculated the nutrient amounts from supplements [19]. This database was developed by identifying the composition of nutritional supplements through searching a medication database, the Korean Ministry of Food and Drug Safety (KMFDS) database, or product distributors [19]. The KCDC has publicly released the nutrient values of calcium, phosphorus, iron, vitamin A, thiamine, riboflavin, niacin, and vitamin $\mathrm{C}$ from the intake of dietary supplements in the KNHANES V-1, 2 (2010-2011). In this study, we developed a database of folate from dietary supplements developed using the same procedure [19].

\section{Statistical analysis}

We applied sampling weight to account for the complex, multistage sampling design of the KNHANES using the SAS survey procedures. Sampling weights were divided by the number of combined data to obtain estimates representative of the Korean population on average from 2010 to 2011 [20]. Descriptive statistics were estimated using PROC SURVEYFREQ for categorical variables and PROC SURVEYMEANS for continuous variables. We identified the proportion (\%) of dietary supplement use of the study population according to the cancer sites of cancer survivors or types of dietary supplements. We presented 10 cancer sites that had enough cancer survivors and combined other cancer sites. We compared the proportion of dietary supplement use between cancer survivors and cancer-free individuals using the Rao-Scott chi-square test. We used PROC SURVEYREG to examine whether nutrient intakes from foods only and total (foods + supplements) differed between cancer survivors and cancer-free individuals. Nutrient intakes were natural logarithm transformed to obtain a normal distribution. We obtained the Least-squares means (LSmeans) and 95\% confidence intervals (95\% CIs) adjusting for age (continuous, years), sex (men, women), energy intake (continuous, $\mathrm{kcal} /$ day), BMI (continuous, $\mathrm{kg} / \mathrm{m}^{2}$ ), and education level (elementary school or below, middle school, high school, college or above). The percent contribution for each participant was calculated by dividing the nutrient intakes from dietary supplements by the total nutrient intakes from foods and dietary supplements. We calculated the mean and standard error (SE) of nutrient intake and of the percent contribution of nutrient intake from dietary supplements to the total intake for cancer survivors and cancer-free individuals. We also identified the proportions of nutrient intakes below the EARs and above the ULs for nutrient intakes of foods and total (foods + supplements) intakes among both cancer survivors and cancer-free individuals. We used the EARs and ULs established by Dietary Reference Intakes for Koreans (KDRIs) 2010 [25]. We compared the proportions below the EARs between cancer survivors and cancer-free individuals using the Rao-Scott chisquare test.

We used PROC SURVEYLOGISTIC to obtain the Odds Ratios (ORs) and 95\% CIs and compare the demographic, lifestyle, and clinical characteristics of supplement users and non-users among cancer survivors. Because we found a sex difference in the characteristics of supplement users, we conducted separate analyses for men and women. In the logistic regression models, we adjusted for age (continuous, years), energy intake (continuous, kcal/day), residential area (rural, urban), and education level (elementary school or below, middle school, high school, college or above). To test for trends, we included the ordinal variable or median values of exposure to the models. To consider the answers on the general questionnaire of dietary supplement use (any supplement use of $>1$ per week over the preceding month; yes/no), we conducted a sensitivity analysis. We found that the results were similar when we excluded those with discrepancies in the responses between the 24-h dietary recalls and the general questionnaire (data not shown). To test for a potential confounding effect of time since cancer diagnosis, we conducted a sensitivity analysis by additionally adjusting for cancer sites with a $>90 \% 5$-year survival rate in 
South Korea (thyroid, breast and prostate cancers), and it resulted in virtually unchanged estimates (data not shown). The level of statistical significance was a $p$-value $<0.05$ in two-sided tests. We used SAS version 9.3 (SAS Institute Inc., Cary, NC, USA) for all statistical analyses.

\section{Results}

We found that $33.3 \%$ of cancer survivors and $22.1 \%$ of cancer-free individuals reported using dietary supplements $(p<0.001)$ (Table 1). Cancer survivors also had a higher rate of dietary supplement use than cancer-free individuals among female $(p=0.001)$ and male $(p=0.04)$. For female cancer survivors, the dietary supplement use rate was the highest in breast cancer survivors (55.9\%), followed by lung (49.6\%), stomach (38.3\%), cervix or corpus uteri (38.1\%), and thyroid cancer survivors (31.7\%). For male cancer survivors, the dietary supplement use rate was the highest in thyroid cancer survivors (31.6\%), followed by bladder (31.5\%), liver (28.5\%), colorectal (26.6\%), and stomach (23.9\%) cancer survivors. The frequency of dietary supplement use without sampling weight is presented in Additional file 1: Table S1.

The major types of dietary supplements consumed by cancer survivors and cancer-free individuals were similar (Table 2). Multi-vitamins and minerals were most commonly used by cancer survivors $(24.6 \%$ among dietary supplement users), followed by vitamin C (18.7\%), omega-3/fish oil (14.8\%), red ginseng (10.7\%), and calcium (4.4\%). Cancer-free individuals also commonly used multi-vitamins and minerals (33.5\% among dietary supplement users), omega-3/fish oil (17.5\%), vitamin C $(13.3 \%)$, red ginseng $(9.0 \%)$, and glucosamine/chondroitin (4.0\%).

We compared nutrient intakes from foods and total (foods + supplements) between cancer and cancer-free individuals (Table 3). For nutrient intakes from foods, cancer survivors had higher amounts of iron $(p=0.04)$, riboflavin $(p=0.01)$, and folate $(p=0.01)$ compared to cancer-free individuals. For total nutrient intakes (foods + supplements), cancer survivors had higher amount of iron $(p=0.04)$, riboflavin $(p=0.03)$, folate $(p=0.02)$, calcium $(p=0.05)$, and vitamin $\mathrm{C}(p=0.01)$ compared to cancerfree individuals. Among dietary supplement users, there were no significant differences between cancer survivors and cancer-free individuals for both nutrient intakes from foods and total (foods + supplements).

Compared to cancer-free individuals, cancer survivors tended to have higher contributions of dietary supplements to total nutrient intakes among all participants (Fig. 1). The contribution of dietary supplements to total nutrient intakes ranged from $0.5 \%$ for phosphorus to $11.6 \%$ for vitamin $C$ among cancer survivors, whereas it ranged from $0.2 \%$ for phosphorus to $7.7 \%$ for vitamin $\mathrm{C}$ among cancer-free individuals. The percent contribution of dietary supplements to total calcium intakes among

Table 1 Dietary supplement use of the study population and according to cancer sites of cancer survivors ${ }^{\mathrm{a}}$

\begin{tabular}{|c|c|c|c|c|c|c|}
\hline & \multicolumn{2}{|l|}{ All } & \multicolumn{2}{|l|}{ Female } & \multicolumn{2}{|l|}{ Male } \\
\hline & Number & Dietary supplement use \% (SE) & Number & Dietary supplement use \% (SE) & Number & Dietary supplement use \% (SE) \\
\hline Cancer-free individuals & 10,387 & $22.1(0.6)$ & 6154 & $26.0(0.7)$ & 4233 & $18.1(0.8)$ \\
\hline Cancer survivors & 400 & $33.3(2.8)$ & 260 & $37.0(3.6)$ & 140 & $26.7(4.7)$ \\
\hline$P$ value ${ }^{b}$ & $<0.001$ & & 0.001 & & 0.04 & \\
\hline \multicolumn{7}{|l|}{ By cancer site } \\
\hline Stomach & 71 & $29.8(6.1)$ & 27 & $38.3(11.4)$ & 44 & $23.9(7.0)$ \\
\hline Cervix or corpus uteri & 67 & $38.1(7.2)$ & 67 & $38.1(7.2)$ & 0 & - \\
\hline Thyroid & 63 & $31.7(6.5)$ & 54 & $31.7(7.1)$ & 9 & $31.6(16.3)$ \\
\hline Breast & 59 & $55.9(7.0)$ & 59 & $55.9(7.0)$ & 0 & - \\
\hline Colorectal & 52 & $22.5(5.9)$ & 27 & $18.5(8.1)$ & 25 & $26.6(8.6)$ \\
\hline Bladder & 14 & $28.7(4.0)$ & 5 & $23.1(0.0)$ & 9 & $31.5(5.9)$ \\
\hline Lung & 13 & $23.0(11.4)$ & 4 & $49.6(28.3)$ & 9 & $15.7(5.4)$ \\
\hline Prostate & 12 & $19.4(7.0)$ & 0 & - & 12 & $19.4(7.0)$ \\
\hline Liver & 11 & $25.8(6.4)$ & 2 & 0.0 & 9 & $28.5(7.1)$ \\
\hline Renal & 9 & 0.0 & 4 & 0.0 & 5 & 0.0 \\
\hline Others $^{c}$ & 46 & $29.2(6.0)$ & 20 & $25.1(12.7)$ & 26 & $32.0(8.4)$ \\
\hline
\end{tabular}

${ }^{a}$ Cancer survivors with multiple cancer sites were counted multiple times; 11 participants had been diagnosed with cancer at two sites, and 3 participants had been diagnosed with cancer at three sites among the 400 cancer survivors

${ }^{\mathrm{b}}$ Rao-Scott chi-square $p$ values were obtained using PROC SURVEYFREQ to compare the proportion of dietary supplement use between cancer survivors and cancer-free individuals

'Others included 19 cancer sites (e.g., larynx, lymphoma, skin, ovarian, and esophageal) 
Table 2 Top 10 dietary supplements commonly used among dietary supplement users ${ }^{\mathrm{a}}$

\begin{tabular}{|c|c|c|c|}
\hline \multicolumn{2}{|l|}{ Cancer survivors $(n=141)$} & \multicolumn{2}{|c|}{ Cancer-free individuals $(n=2651)$} \\
\hline Supplement type & $\%(\mathrm{SE})$ & Supplement type & $\%(\mathrm{SE})$ \\
\hline Multi-vitamin/minerals & $24.6(4.5)$ & Multi-vitamin/minerals & $33.5(1.3)$ \\
\hline Vitamin $C^{b}$ & $18.7(4.8)$ & Omega-3/fish oil & $17.5(1.0)$ \\
\hline Omega-3/fish oil & $14.8(3.8)$ & Vitamin $C^{b}$ & $13.3(0.9)$ \\
\hline Red ginseng & $10.7(4.3)$ & Red ginseng & $9.0(0.8)$ \\
\hline Calcium $^{c}$ & $4.4(2.2)$ & $\begin{array}{l}\text { Glucosamine/ } \\
\text { Chondroitin }\end{array}$ & $4.0(0.5)$ \\
\hline Yeast & $4.2(3.0)$ & Calcium $^{c}$ & $3.7(0.4)$ \\
\hline $\begin{array}{l}\text { Glucosamine/ } \\
\text { Chondroitin }\end{array}$ & $2.4(1.0)$ & Evening primrose oil & $2.3(0.4)$ \\
\hline Garlic extract & $1.9(1.3)$ & Vitamin $\mathrm{Bs}^{\mathrm{d}}$ & $1.1(0.2)$ \\
\hline $\begin{array}{l}\text { Mycelial culture extract } \\
\text { from phellinus linteus }\end{array}$ & $1.5(1.5)$ & Vitamin $\mathrm{E}$ & $1.1(0.2)$ \\
\hline Octacosanol & $1.0(1.0)$ & Vitamin A/Beta-carotene & $0.9(0.2)$ \\
\hline
\end{tabular}

${ }^{\mathrm{a} C a n c e r}$ survivors who used more than one dietary supplement were counted multiple times

${ }^{b}$ Vitamin C and Vitamin C (major component) + Vitamin E

${ }^{\mathrm{c} C a l c i u m,}$ Calcium (major component) + Vitamin D, and Calcium (major component) + Vitamin D + Magnesium

${ }^{\mathrm{d}}$ Thiamine, Vitamin B6, Folate, Vitamin B12, Folate + Vitamin B12, and Vitamin B complex cancer survivors $(4.7 \%)$ was 2.6 times higher than that among cancer-free individuals (1.8\%). Calcium and vitamin $C$ showed a relatively higher difference of the percent contribution of dietary supplements to total intakes between cancer survivors and cancer-free individuals than other nutrients. Among supplement users, the contribution of dietary supplement to total nutrient intakes were similar between cancer survivors and cancer-free individuals, except for calcium (Fig. 2).

We found that a higher proportion of cancer survivors consumed thiamin $(p=0.02)$ and niacin $(p=0.03)$ from foods and supplements below the EARs compared to cancer-free individuals (Table 4). Among dietary supplement users, cancer survivors had a higher proportion of individuals whose intakes of vitamin $\mathrm{A}(p=0.03)$, thia$\min (p=0.01)$, riboflavin $(p=0.01)$, and folate $(p=0.01)$ from foods and supplements were below the EARs compared to cancer-free individuals. Overall, both cancer survivors and cancer-free individuals showed that more than $60 \%$ consumed calcium from foods and supplements below the EARs. The proportions of study population who consumed nutrients below the EARs from foods were reduced after the addition of nutrient intakes from dietary supplements. Among all participants, the

Table 3 Nutrient intakes from food and total (foods and supplements) among cancer survivors and cancer-free individuals

\begin{tabular}{|c|c|c|c|c|c|c|}
\hline \multirow[t]{2}{*}{ LS means $(95 \% \mathrm{Cl})^{\mathrm{a}}$} & \multicolumn{2}{|l|}{ Food only } & \multirow{2}{*}{$\begin{array}{l}P \\
\text { value }^{\mathrm{b}}\end{array}$} & \multicolumn{2}{|l|}{ Foods + Supplements } & \multirow{2}{*}{$\begin{array}{l}P \\
\text { value }^{k}\end{array}$} \\
\hline & Cancer survivors & Cancer-free individuals & & Cancer survivors & Cancer-free individuals & \\
\hline All (n) & 400 & 10,387 & & 400 & 10,387 & \\
\hline Calcium (mg/d) & 437.6 (400.9-477.6) & $413.6(407-420.2)$ & 0.21 & $465.0(425.1-508.7)$ & $424.8(418.0-431.7)$ & 0.05 \\
\hline Phosphorus (mg/d) & $1083.0(1040.9-1126.9)$ & $1056.9(1049.7-1064.0)$ & 0.23 & $1088.0(1045.4-1132.2)$ & $1060.0(1052.9-1067.2)$ & 0.21 \\
\hline Iron (mg/d) & $13.3(12.4-14.3)$ & $12.3(12.1-12.5)$ & 0.04 & $13.9(12.8-15.0)$ & $12.8(12.6-13.0)$ & 0.04 \\
\hline Vitamin A ( $\mu \mathrm{g} R E / d)$ & $552.3(491.8-620.2)$ & $531.0(516.3-546.2)$ & 0.51 & $582.9(517.7-656.4)$ & $560.9(544.7-577.6)$ & 0.53 \\
\hline Thiamine (mg/d) & $1.2(1.1-1.2)$ & $1.1(1.1-1.1)$ & 0.09 & $1.4(1.3-1.5)$ & $1.3(1.3-1.3)$ & 0.35 \\
\hline Riboflavin (mg/d) & $1.1(1.0-1.1)$ & $1.0(1.0-1.0)$ & 0.01 & $1.2(1.1-1.3)$ & $1.1(1.1-1.2)$ & 0.03 \\
\hline Niacin (mg/d) & $14.9(14.3-15.5)$ & $14.4(14.3-14.5)$ & 0.10 & $16.4(15.4-17.4)$ & $15.6(15.4-15.8)$ & 0.11 \\
\hline Folate ( $\mu \mathrm{g}$ DFE/d) & 335.7 (316.7-355.8) & $308.6(304.4-312.8)$ & 0.01 & 358.5 (334.8-383.9) & $330.9(325.8-336.2)$ & 0.02 \\
\hline Vitamin C (mg/d) & $87.9(77.4-99.8)$ & $77.8(76.1-79.6)$ & 0.06 & $111.5(96.4-128.9)$ & $91.4(89.0-93.9)$ & 0.01 \\
\hline Among users (n) & 141 & 2651 & & 141 & 2651 & \\
\hline Calcium (mg/d) & 443.5 (396.3-496.5) & $440.2(425.9-454.9)$ & 0.89 & $532.5(470.4-602.7)$ & $492.7(475.7-510.4)$ & 0.22 \\
\hline Phosphorus (mg/d) & $1080.4(1022.9-1141.2)$ & $1092.9(1078.7-1107.4)$ & 0.68 & $1096.3(1037.9-1158.1)$ & $1107.7(1093.0-1122.4)$ & 0.71 \\
\hline Iron (mg/d) & $13.7(12.5-15.1)$ & $13.1(12.6-13.5)$ & 0.34 & $15.5(13.7-17.4)$ & $15.2(14.6-15.8)$ & 0.81 \\
\hline Vitamin A ( $\mu \mathrm{g} R E / d)$ & $543.9(468.6-631.2)$ & $567.0(539.2-596.2)$ & 0.59 & $645.3(554.4-751.1)$ & 718.8 (678.5-761.4) & 0.17 \\
\hline Thiamine (mg/d) & $1.2(1.1-1.3)$ & $1.1(1.1-1.2)$ & 0.13 & $2.2(1.7-2.8)$ & $2.4(2.2-2.5)$ & 0.56 \\
\hline Riboflavin (mg/d) & $1.0(1.0-1.1)$ & $1.0(1.0-1.1)$ & 0.92 & $1.8(1.5-2.2)$ & $1.9(1.8-2.0)$ & 0.63 \\
\hline Niacin (mg/d) & $14.7(13.8-15.6)$ & $14.7(14.4-14.9)$ & 0.99 & $20.1(17.4-23.1)$ & $21.0(20.2-21.9)$ & 0.54 \\
\hline Folate ( $\mu \mathrm{g}$ DFE/d) & $333.3(306.1-362.9)$ & $321.3(313.8-329)$ & 0.41 & $409(359.3-465.7)$ & $434.8(420.4-449.8)$ & 0.37 \\
\hline Vitamin C (mg/d) & $86.0(73.7-100.4)$ & $82.8(79.6-86.2)$ & 0.64 & $185.7(145.2-237.3)$ & 170.6 (160.4-181.4) & 0.51 \\
\hline
\end{tabular}

Abbreviations: LS means Least squares means, 95\% CI 95\% confidence interval, $R E$ retinol equivalent, DFE dietary folate equivalent

a Least square means adjusted for age (continuous, years), sex (men, women), energy intake (continuous, $\mathrm{kcal} /$ day), body mass index (continuous, $\left.\mathrm{kg} / \mathrm{m}^{2}\right)$, and education level (elementary school or below, middle school, high school, and college or above) were obtained using PROC SURVEYREG

${ }^{\mathrm{b}} P$ values were obtained based on the Wald's $\mathrm{F}$ test 


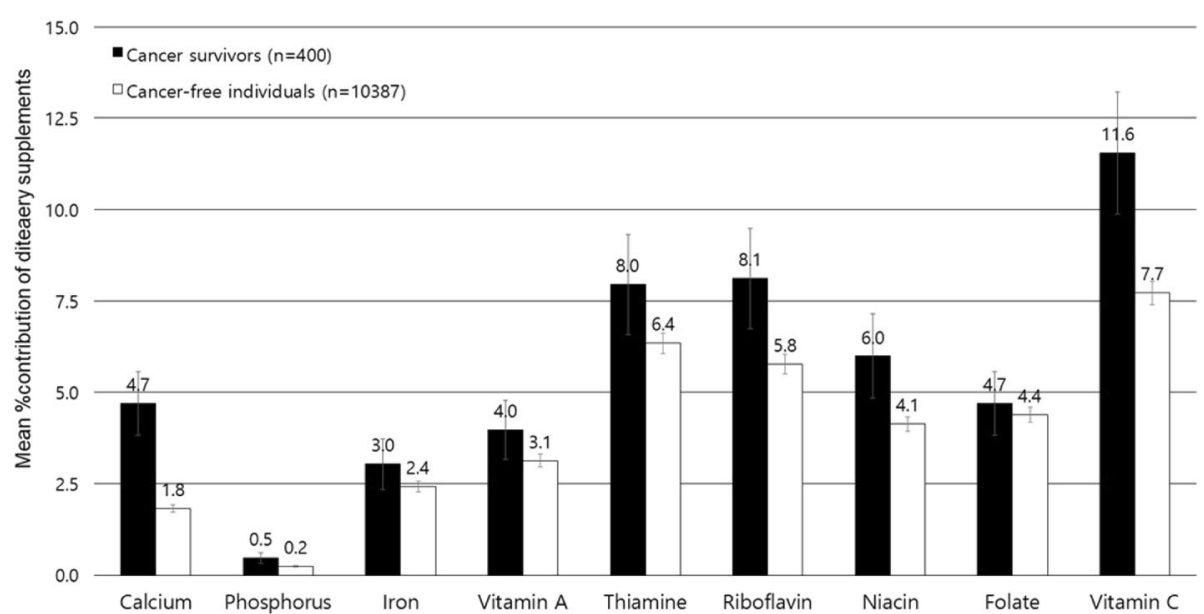

Fig. 1 The percent contribution from dietary supplements (Mean \pm SE) to total nutrient intakes among overall participants (400 cancer survivors and 10,387 cancer-free individuals)

proportions of cancer survivors who consumed nutrients below the EARs ranged from $31.6 \%$ (niacin) to $49.1 \%$ (riboflavin) for six nutrients (vitamin A, thiamin, riboflavin, niacin, folate, and vitamin $C$ ) from foods and supplements; for cancer-free individuals, more than $30 \%$, but less than $50 \%$, consumed levels of vitamin A, riboflavin, folate, and vitamin $\mathrm{C}$ below the EARs from foods and supplements. Among dietary supplement users, the proportions of consumption below EARs were reduced after adding nutrient intake from supplement to that from foods only by an average of $24.3 \%$ among cancer survivors and $30.3 \%$ among cancer-free individuals with the highest reduction for vitamin $\mathrm{C}$.

A small proportion of cancer survivors consumed nutrient intakes exceeding ULs, even after the addition of intakes from supplements (Additional file 2: Table S2). The proportions of consumption above ULs for nutrient intakes from foods and supplements ranged from $0.3 \%$ for phosphorus to $3.3 \%$ for iron among cancer survivors and ranged $0.2 \%$ for calcium to $2.9 \%$ for vitamin A among cancer-free individuals. Among dietary supplement users, the proportions of consumption above ULs were less than $4 \%$ among cancer survivors and less than $6 \%$ among cancer-free individuals.

We examined whether demographic, lifestyle, and clinical factors were associated with dietary supplement use among cancer survivors (Table 5). Among female cancer survivors, dietary supplement use was associated with education level: the ORs (95\% CIs) were 4.75 (95\% CI = 1.66-13.56) for middle school vs. elementary school or below and 4.51 (95\% CI $=1.69-12.06)$ for high school vs. elementary school or below. Female cancer survivor supplement users were more likely to engage in moderate physical activity $(\mathrm{OR}=3.95 ; 95 \% \mathrm{CI}=1.16-13.44$ for yes vs. no) and were less likely to consume vegetables $(\mathrm{OR}=$ $0.81 ; 95 \% \mathrm{CI}=0.70-0.94$ for one increment in one

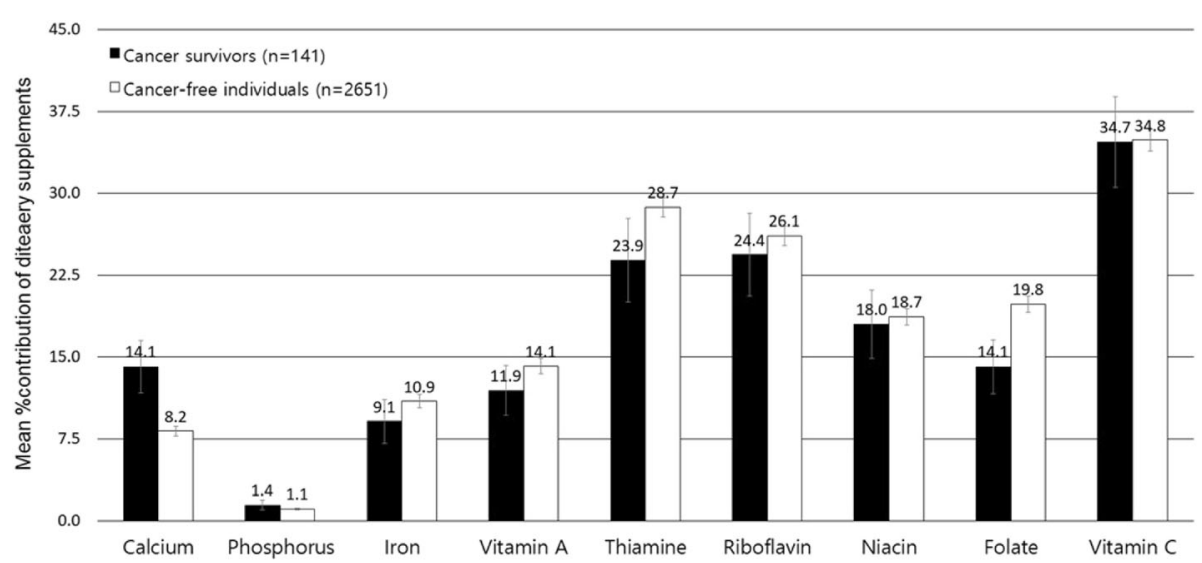

Fig. 2 The percent contribution from dietary supplements (Mean \pm SE) to total nutrient intakes among dietary supplement users (141 cancer survivors and 2651 cancer-free individuals) 
Table 4 The proportions of below estimated average requirement (EAR) among cancer survivors and cancer-free individuals

\begin{tabular}{|c|c|c|c|c|c|c|c|c|}
\hline \multirow[t]{3}{*}{ Nutrient } & \multicolumn{3}{|c|}{ Cancer survivors } & \multicolumn{3}{|c|}{ Cancer-free individuals } & \multirow[t]{3}{*}{$P$ value $^{b}$} & \multirow[t]{3}{*}{$P$ value ${ }^{c}$} \\
\hline & \multicolumn{2}{|c|}{ \% Below EAR (SE) } & \multirow[b]{2}{*}{$\%$ Change $^{a}$} & \multicolumn{2}{|c|}{ \% Below EAR (SE) } & \multirow[b]{2}{*}{$\%$ Change $^{a}$} & & \\
\hline & Food only & Foods and supplements & & Food only & Foods and supplements & & & \\
\hline All (n) & 400 & & & 10,387 & & & & \\
\hline Calcium (mg/d) & $64.2(3.2)$ & $61.2(3.1)$ & 4.7 & $64.7(0.7)$ & $63.0(0.7)$ & 2.6 & 0.88 & 0.57 \\
\hline Phosphate (mg/d) & $9.8(1.8)$ & $9.7(1.8)$ & 1.0 & $7.9(0.3)$ & $7.9(0.3)$ & 0.0 & 0.27 & 0.28 \\
\hline Iron (mg/d) & $18.6(2.5)$ & $17.2(2.5)$ & 7.5 & $22.1(0.5)$ & $21.1(0.5)$ & 4.5 & 0.20 & 0.15 \\
\hline Vitamin A ( $\mu \mathrm{g}$ RE/d) & $44.0(3.1)$ & $40.3(3.1)$ & 8.4 & $37.3(0.7)$ & $35.6(0.7)$ & 4.6 & 0.03 & 0.11 \\
\hline Thiamin (mg/d) & $40.4(3.2)$ & $36.3(3.2)$ & 10.2 & $32.1(0.6)$ & $29.3(0.6)$ & 8.7 & 0.01 & 0.02 \\
\hline Riboflavin (mg/d) & $56.3(3.1)$ & $49.1(3.1)$ & 12.8 & $49.4(0.7)$ & $45.6(0.7)$ & 7.7 & 0.02 & 0.25 \\
\hline Niacin (mg/d) & $35.4(3.0)$ & $31.6(2.7)$ & 10.7 & $27.8(0.6)$ & $25.7(0.6)$ & 7.6 & 0.01 & 0.03 \\
\hline Folate ( $\mu \mathrm{g} / \mathrm{d})$ & $46.8(3.3)$ & $43.5(3.4)$ & 7.1 & $47.0(0.7)$ & $43.7(0.6)$ & 7.0 & 0.93 & 0.94 \\
\hline Vitamin C (mg/d) & $42.2(3.2)$ & $35.9(3.0)$ & 14.9 & $42.3(0.7)$ & $37.8(0.7)$ & 10.6 & 0.96 & 0.51 \\
\hline Among users (n) & 141 & & & 2651 & & & & \\
\hline Calcium (mg/d) & $68.7(5.0)$ & $59.5(5.2)$ & 13.4 & $58.7(1.3)$ & $50.8(1.3)$ & 13.5 & 0.06 & 0.11 \\
\hline Phosphate (mg/d) & $7.0(2.7)$ & $6.7(2.7)$ & 4.3 & $5.7(0.6)$ & $5.5(0.6)$ & 3.5 & 0.63 & 0.64 \\
\hline Iron (mg/d) & 19.5 (4.6) & $15.1(4.2)$ & 22.6 & $18.2(1.0)$ & $13.6(0.9)$ & 25.3 & 0.79 & 0.71 \\
\hline Vitamin A ( $\mu \mathrm{g} R E / d)$ & $46.4(5.4)$ & $35.4(5.1)$ & 23.7 & $33.0(1.2)$ & $24.9(1.1)$ & 24.6 & 0.01 & 0.03 \\
\hline Thiamin (mg/d) & $42.9(5.5)$ & $30.7(5.5)$ & 28.4 & $30.8(1.2)$ & $18.1(1.0)$ & 41.2 & 0.02 & 0.01 \\
\hline Riboflavin (mg/d) & $63.7(5.3)$ & $42.1(5.3)$ & 33.9 & $45.3(1.3)$ & $28.2(1.0)$ & 37.8 & 0.001 & 0.01 \\
\hline Niacin (mg/d) & $36.4(5.8)$ & $25.0(5.0)$ & 31.3 & $26.4(1.1)$ & $16.6(0.9)$ & 37.1 & 0.06 & 0.05 \\
\hline Folate $(\mu \mathrm{g} / \mathrm{d})$ & $52.7(5.5)$ & $42.8(5.6)$ & 18.8 & $43.3(1.3)$ & $28.4(1.1)$ & 34.4 & 0.10 & 0.01 \\
\hline Vitamin C (mg/d) & $44.6(5.4)$ & $25.7(4.5)$ & 42.4 & $38.6(1.3)$ & $18.4(0.9)$ & 52.3 & 0.27 & 0.07 \\
\hline
\end{tabular}

Abbreviations: $R E$ retinol equivalent, DFE dietary folate equivalents

${ }^{a}$ Calculated by dividing subtracted values (\% below EAR from foods only - foods and supplements) by \% below EAR from foods only

${ }^{\mathrm{b}}$ Rao-Scott chi-square $p$ values were obtained using PROC SURVEYFREQ to compare the proportion of below EAR from foods between survivors and

cancer-free individuals

${ }^{c}$ Rao-Scott chi-square $p$ values were obtained using PROC SURVEYFREQ to compare the proportion of below EAR from foods and supplements between survivors and cancer-free individuals

serving size of vegetable intake) compared to non-users. Among male cancer survivors, supplement users were less likely to live in rural areas $(\mathrm{OR}=0.26 ; 95 \% \mathrm{CI}=$ 0.08-0.84 for rural vs. urban) and were more likely to have lower total energy consumption $(\mathrm{OR}=0.93$; 95\% $\mathrm{CI}=0.88-0.99$ for $100 \mathrm{kcal} / \mathrm{d}$ increment in energy intake) compared to non-users. Moreover, among male cancer survivors, supplement users were more likely to be never alcohol drinkers $(\mathrm{OR}=7.88 ; 95 \% \mathrm{CI}=1.45-42.82$ for never alcohol drinkers vs. ever alcohol drinkers) compared to non-users. We also examined the associations between dietary supplement use and blood levels of fasting blood glucose, total and high-density lipoprotein (HDL) cholesterol, triglycerides, and 25-hydroxyvitamin D3 (25(OH) D3). The use of dietary supplements was not significantly associated with these blood markers except for 25(OH) D3 among female cancer survivors; dietary supplement users tended to have higher $25(\mathrm{OH})$ D3 levels compared to non-users $(\mathrm{OR}=1.27 ; 95 \% \mathrm{CI}=1.01-1.60$ for a $5 \mathrm{ng} / \mathrm{mL}$ increment in $25(\mathrm{OH}) \mathrm{D} 3)$.

\section{Discussion}

We aimed to examine the use of dietary supplement, the amount of nutrients consumed from foods and supplements, and their nutritional contribution to total intakes among cancer survivors and cancer-free individuals. This nationwide study found that Korean cancer survivors had a higher prevalence of dietary supplement use than cancer-free individuals. The contribution of nutrient intakes from dietary supplements to total nutrient intakes was higher among cancer survivors than cancerfree individuals. We also aimed to identify the factors associated with dietary supplement use among cancer survivors, and we found that education level above elementary school, moderate physical activity, low vegetable intake, and high circulating vitamin $\mathrm{D}$ levels were associated with dietary supplement use among female cancer survivors, whereas living in an urban area, no history of alcohol consumption, and low energy intake were associated with dietary supplement use among male cancer survivors. 
Table 5 Odds ratios (ORs) and 95\% confidence intervals $(\mathrm{Cls})^{a}$ for supplement users vs non-users $(n=400)$

\begin{tabular}{|c|c|c|c|c|c|c|c|c|}
\hline \multirow[t]{3}{*}{ Characteristics } & \multicolumn{4}{|c|}{ Female cancer survivors } & \multicolumn{4}{|c|}{ Male cancer survivors } \\
\hline & \multirow[t]{2}{*}{ Number } & $\begin{array}{l}\text { Non-use } \\
(n=160)\end{array}$ & $\begin{array}{l}\text { Supplement } \\
\text { use }(n=100)\end{array}$ & $\begin{array}{l}\text { Any supplement } \\
\text { use vs. non-use }\end{array}$ & Number & $\begin{array}{l}\text { Non-use } \\
(n=99)\end{array}$ & $\begin{array}{l}\text { Supplement } \\
\text { use }(n=41)\end{array}$ & $\begin{array}{l}\text { Any supplement } \\
\text { use vs. non-use }\end{array}$ \\
\hline & & $\%(\mathrm{SE})$ & $\%(\mathrm{SE})$ & OR $(95 \% \mathrm{Cl})$ & & $\%(\mathrm{SE})$ & $\%(\mathrm{SE})$ & OR $(95 \% \mathrm{Cl})$ \\
\hline \multicolumn{9}{|l|}{ Age (years) } \\
\hline $19-59$ & 134 & $56.7(4.8)$ & $68.6(5.3)$ & Reference & 37 & $49.3(6.1)$ & $41.4(10.8)$ & Reference \\
\hline $60-69$ & 74 & $22.9(3.9)$ & $19.5(4.3)$ & $0.95(0.43-2.11)$ & 49 & $24.8(5.1)$ & $28.3(7.6)$ & $1.97(0.53-7.30)$ \\
\hline$\geq 70$ & 52 & $20.4(3.8)$ & $11.9(3.4)$ & $0.85(0.28-2.57)$ & 54 & $25.8(4.2)$ & $30.4(8.4)$ & $1.35(0.36-5.05)$ \\
\hline$P$ for trend & & & & 0.78 & & & & 0.58 \\
\hline \multicolumn{9}{|l|}{ Marital status } \\
\hline Married & 252 & $93.5(2.9)$ & $97.8(2.1)$ & Reference & 134 & $88.0(5.4)$ & $89.8(7.5)$ & Reference \\
\hline Not married & 8 & $6.5(2.9)$ & $2.2(2.1)$ & $0.24(0.02-2.82)$ & 6 & $12.0(5.4)$ & $10.2(7.5)$ & $0.61(0.05-7.11)$ \\
\hline \multicolumn{9}{|l|}{ Residential area } \\
\hline Urban & 202 & $77.0(4.0)$ & $79.6(6.1)$ & Reference & 99 & $65.8(5.9)$ & $86.3(5.7)$ & Reference \\
\hline Rural & 58 & $23.0(4.0)$ & $20.4(6.1)$ & $1.04(0.49-2.17)$ & 41 & $34.2(5.9)$ & $13.7(5.7)$ & $0.26(0.08-0.84)$ \\
\hline \multicolumn{9}{|l|}{ Education level } \\
\hline Elementary school or below & 101 & $42.4(4.6)$ & $20.2(4.3)$ & Reference & 45 & $27.8(4.5)$ & $18.1(6.3)$ & Reference \\
\hline Middle school & 48 & $15.4(3.2)$ & $25.5(6.0)$ & $4.75(1.66-13.56)$ & 16 & $5.9(2.3)$ & $15.6(6.5)$ & $5.08(0.91-28.55)$ \\
\hline High school & 70 & $24.0(4.5)$ & $36.1(6.0)$ & $4.51(1.69-12.06)$ & 41 & $38.2(6.7)$ & $18.4(6.4)$ & $0.73(0.18-2.87)$ \\
\hline College or above & 41 & $18.2(4.1)$ & $18.2(4.9)$ & $3.18(0.92-11.04)$ & 38 & $28.0(6.0)$ & $47.8(10.3)$ & $3.50(0.78-15.75)$ \\
\hline$P$ for trend & & & & 0.07 & & & & 0.15 \\
\hline \multicolumn{9}{|l|}{ Occupation $^{\text {b }}$} \\
\hline Unemployed & 173 & $63.9(4.8)$ & $57.2(6.9)$ & Reference & 78 & $53.0(6.3)$ & $37.5(8.8)$ & Reference \\
\hline Employed & 87 & $36.1(4.8)$ & $42.8(6.9)$ & $1.30(0.65-2.60)$ & 61 & $47.0(6.3)$ & $62.5(8.8)$ & $2.57(0.98-6.75)$ \\
\hline \multicolumn{9}{|c|}{ Equalized monthly household income ${ }^{b}$} \\
\hline Low & 67 & $26.3(4.3)$ & $18.6(4.5)$ & Reference & 46 & $27.4(4.9)$ & $29.6(8.3)$ & Reference \\
\hline Mid-low & 62 & $25.5(4.7)$ & $25.3(5.7)$ & $1.14(0.45-2.88)$ & 35 & $24.7(5.9)$ & $26.0(11.0)$ & $1.49(0.43-5.12)$ \\
\hline Mid-high & 59 & $26.2(4.4)$ & $18.9(5.1)$ & $0.87(0.34-2.25)$ & 31 & $24.6(5.6)$ & $23.2(9.0)$ & $0.67(0.19-2.33)$ \\
\hline High & 68 & $22.1(4.5)$ & $37.2(6.1)$ & $1.71(0.66-4.47)$ & 26 & $23.3(5.4)$ & $21.2(7.3)$ & $1.02(0.23-4.47)$ \\
\hline$P$ for trend & & & & 0.33 & & & & 0.76 \\
\hline Body weight $(\mathrm{kg})^{\mathrm{b}, \mathrm{c}}$ & 260 & $56.4 \pm 0.9$ & $59.1 \pm 1.3$ & $1.02(0.99-1.06)$ & 139 & $65.6 \pm 1.8$ & $65 \pm 2.0$ & $1.00(0.95-1.05)$ \\
\hline \multicolumn{9}{|l|}{ Body mass index ${ }^{b}$} \\
\hline$<18.5$ & 11 & $6.0(2.6)$ & $5.3(3.5)$ & $1.54(0.33-7.11)$ & 10 & $7.9(4.1)$ & $6.4(3.4)$ & $1.45(0.21-10.18)$ \\
\hline $18.5-22.9$ & 96 & $44.2(5.2)$ & $32.7(5.9)$ & Reference & 68 & $45.3(6.0)$ & $52.8(10.6)$ & Reference \\
\hline $23-24.9$ & 59 & $20.2(3.5)$ & $20.0(4.7)$ & $1.51(0.68-3.36)$ & 29 & $25.2(5.1)$ & $14.4(5.8)$ & $0.43(0.10-1.93)$ \\
\hline$\geq 25$ & 94 & $29.6(4.4)$ & $41.9(6.3)$ & $2.04(0.93-4.50)$ & 32 & $21.6(5.5)$ & $26.4(9.0)$ & $1.62(0.41-6.42)$ \\
\hline$P$ for trend & & & & 0.17 & & & & 0.93 \\
\hline Waist circumference $(\mathrm{cm})^{\mathrm{b}, \mathrm{c}}$ & 259 & $78.3 \pm 0.9$ & $80.6 \pm 1.4$ & $1.03(0.99-1.07)$ & 139 & $82.9 \pm 1.3$ & $81.4 \pm 1.6$ & $0.98(0.93-1.04)$ \\
\hline \multicolumn{9}{|l|}{ Vigorous physical activity } \\
\hline No & 231 & $88.1(3.5)$ & $87.6(4.6)$ & Reference & 112 & $78.8(5.0)$ & $73.7(11.7)$ & Reference \\
\hline Yes & 27 & $11.9(3.5)$ & $12.4(4.6)$ & $1.00(0.30-3.36)$ & 27 & $21.2(5.0)$ & $26.3(11.7)$ & $0.95(0.26-3.56)$ \\
\hline \multicolumn{9}{|l|}{ Moderate physical activity, be } \\
\hline No & 243 & $96.8(1.2)$ & $89.6(5.1)$ & Reference & 121 & $84.1(4.7)$ & $82.2(10.9)$ & Reference \\
\hline Yes & 16 & $3.2(1.2)$ & $10.4(5.1)$ & $3.95(1.16-13.44)$ & 19 & $15.9(4.7)$ & $17.8(10.9)$ & $1.51(0.32-7.03)$ \\
\hline
\end{tabular}


Table 5 Odds ratios (ORs) and 95\% confidence intervals (Cls) for supplement users vs non-users $(n=400)$ (Continued)

\begin{tabular}{|c|c|c|c|c|c|c|c|c|}
\hline \multirow[t]{3}{*}{ Characteristics } & \multicolumn{4}{|c|}{ Female cancer survivors } & \multicolumn{4}{|c|}{ Male cancer survivors } \\
\hline & \multirow[t]{2}{*}{ Number } & $\begin{array}{l}\text { Non-use } \\
(n=160)\end{array}$ & $\begin{array}{l}\text { Supplement } \\
\text { use }(n=100)\end{array}$ & $\begin{array}{l}\text { Any supplement } \\
\text { use vs. non-use }\end{array}$ & Number & $\begin{array}{l}\text { Non-use } \\
(n=99)\end{array}$ & $\begin{array}{l}\text { Supplement } \\
\text { use }(n=41)\end{array}$ & $\begin{array}{l}\text { Any supplement } \\
\text { use vs. non-use }\end{array}$ \\
\hline & & $\%(\mathrm{SE})$ & $\%(\mathrm{SE})$ & OR $(95 \% \mathrm{Cl})$ & & $\%$ (SE) & $\%(\mathrm{SE})$ & OR $(95 \% \mathrm{Cl})$ \\
\hline \multicolumn{9}{|l|}{ Smoking status $^{a}$} \\
\hline Never smoker & 240 & $94.0(2.4)$ & $90.0(3.5)$ & Reference & 24 & $17.3(5.4)$ & $35.0(11.1)$ & Reference \\
\hline Ever smoker & 19 & $6.0(2.4)$ & $10.0(3.5)$ & $2.15(0.79-5.81)$ & 116 & $82.7(5.4)$ & $65.0(11.1)$ & $0.35(0.10-1.17)$ \\
\hline \multicolumn{9}{|l|}{ Alcohol intake ${ }^{a}$} \\
\hline Ever drinker & 173 & $73.1(4.2)$ & $66.3(6.2)$ & Reference & 129 & $97.3(1.6)$ & $81.2(10.8)$ & Reference \\
\hline Never drinker & 86 & $26.9(4.2)$ & $33.7(6.2)$ & $1.54(0.66-3.59)$ & 11 & $2.7(1.6)$ & $18.8(10.8)$ & $7.88(1.45-42.82)$ \\
\hline Energy intakes $(100 \mathrm{kcal} / \text { day })^{c}$ & 260 & $16.7 \pm 0.7$ & $16.0 \pm 0.6$ & $0.96(0.91-1.02)$ & 140 & $22.2 \pm 1.4$ & $19.0 \pm 1.1$ & $0.93(0.88-0.99)$ \\
\hline \multicolumn{9}{|l|}{ Food intakes (serving/day) ${ }^{\mathrm{b}, \mathrm{c}}$} \\
\hline Vegetables & 253 & $4.5 \pm 0.3$ & $3.9 \pm 0.2$ & $0.81(0.70-0.94)$ & 131 & $4.2 \pm 0.2$ & $4.6 \pm 0.5$ & $1.12(0.87-1.46)$ \\
\hline Fruits & 253 & $1.3 \pm 0.1$ & $1.4 \pm 0.08$ & $0.90(0.64-1.27)$ & 131 & $0.9 \pm 0.06$ & $1.1 \pm 0.3$ & $1.77(0.82-3.81)$ \\
\hline Red and processed meat & 253 & $0.2 \pm 0.02$ & $0.3 \pm 0.04$ & $2.08(0.45-9.56)$ & 131 & $0.3 \pm 0.03$ & $0.3 \pm 0.06$ & $3.58(0.18-70.93)$ \\
\hline \multicolumn{9}{|l|}{ Time since cancer diagnosis b $^{\mathrm{b}} \mathrm{f}$} \\
\hline Less than 5 years & 118 & $50.7(5.2)$ & $47.5(6.0)$ & Reference & 74 & $50.8(6.1)$ & $41.6(9.5)$ & Reference \\
\hline 5 years or more & 141 & $49.3(5.2)$ & $52.5(6.0)$ & $1.44(0.71-2.89)$ & 66 & $49.2(6.1)$ & $58.4(9.5)$ & $1.35(0.53-3.43)$ \\
\hline \multicolumn{9}{|l|}{ Chronic morbidity $^{9}$} \\
\hline No & 122 & $52.4(5.0)$ & $62.9(5.5)$ & Reference & 70 & $62.4(5.9)$ & $55.8(10.0)$ & Reference \\
\hline Yes & 138 & $47.6(5.0)$ & $37.1(5.5)$ & $0.93(0.40-2.16)$ & 70 & $37.6(5.9)$ & $44.2(10.0)$ & $1.19(0.38-3.70)$ \\
\hline
\end{tabular}

${ }^{a}$ Models were adjusted for age (years, continuous), energy intakes (kcal/day, continuous), residential area (rural, urban), and education level (elementary school or below, middle school, high school, and college or above)

${ }^{\mathrm{b}}$ Total number of participants was not equal to 260 for women or 140 for men because of some did not provide the relevant information

cContinuous variables are expressed as Mean \pm SE

${ }^{\mathrm{d} V i g o r o u s ~ p h y s i c a l ~ a c t i v i t y ~ w a s ~ d e f i n e d ~ a s ~ a t ~ l e a s t ~} 20$ min of vigorous-intensity activity on $\geq 3$ days per week

eModerate physical activity was defined as at least $30 \mathrm{~min}$ of moderate-intensity activity on $\geq 5$ days per week

fTime since cancer diagnosis was calculated by subtracting the age at the first diagnosis of cancer from the current age

${ }_{9}^{9}$ Participants were categorized into yes if they answered to ever have hypertension, dyslipidemia, stroke, myocardial infraction and/or angina, osteoarthritis and/or rheumatoid arthritis, and diabetes mellitus

Our study showed that $33.3 \%$ of cancer survivors reported the use of dietary supplements compared to $22.1 \%$ of cancer-free individuals. A systematic review of dietary supplement use among cancer survivors reported that $64-81 \%$ of cancer survivors used any vitamin or mineral supplements, which may be a higher proportion compared to general US adults (52\%) [5]. However, previous studies that compared dietary supplement use between cancer survivors and cancer-free individuals are inconsistent [26-29]. The 2001 California Health Information Survey and the 2003 Complementary and Alternative Medicine Supplement to the California Health Information Survey found that cancer survivors were more likely to use vitamin supplements, but not minerals, herbs, and other natural products, compared to cancer-free individuals [26]. The 1987 and 1992 National Health Interview Surveys (NHIS) and Vitamins and Lifestyle (VITAL) study found no differences in the dietary supplement use rate between cancer survivors and cancer-free individuals but found that relatively high proportions of cancer survivors and cancer-free individuals used multivitamins (approximately
$50 \%$ in the NHIS and over $70 \%$ in the VITAL study) $[27,28]$. In the databank and biorepository (DBBR), cancer patients were less likely to use dietary supplements than cancer-free individuals [29].

Several US epidemiologic studies reported that multivitamins and minerals have reported as the most commonly consumed supplement type in both cancer survivors [5] and general population [30, 31]. Also, antioxidants, calcium/vitamin D, herbal/botanical, and fatty acids have reported as supplement types commonly consumed by US cancer survivors $[10,32,33]$. Consistent with these studies, we also found that multi-vitamins and minerals was the most commonly consumed supplement types in both cancer survivors (24.6\% of dietary supplement users) and cancer-free individuals (33.5\%). Other supplements consumed commonly were vitamin $\mathrm{C}$, omega-3/fish oil, red ginseng, and calcium among cancer survivors and omega-3/fish oil, vitamin C, red ginseng, and glucosamine/ chondroitin among cancer-free individuals in our study.

Limited studies have compared the amount of nutrients calculated from foods and supplements between cancer 
survivors and cancer-free individuals. The use of antioxidant dietary supplements in breast cancer survivors from the Women's Healthy Eating and Living (WHEL) and general female population from the Olestra Post-Marketing Surveillance Study (OPMSS) were compared in the conference of "Free Radicals: The Pros and Cons of Antioxidants" [34]. The presenters reported that, among dietary supplement users, the median intakes of vitamin $\mathrm{C}$ and betacarotene from dietary supplements were similar between the two groups, but the median supplemental vitamin $\mathrm{E}$ intakes were much higher among cancer survivors $(268 \mathrm{mg} / \mathrm{d})$ in the WHEL study than cancer-free individuals $(34 \mathrm{mg} / \mathrm{d})$ in the OPMSS study [34]. In our study, among overall participants, cancer survivors consumed higher amounts of calcium and vitamin $\mathrm{C}$ than cancerfree individuals after the addition of nutrient intakes from dietary supplements. Among dietary supplement users, however, nutrient intakes from foods only and total (foods + supplements) were similar between cancer survivors and cancer-free individuals.

We found that the percent contribution of dietary supplements to total nutrient intakes among cancer survivors who consumed dietary supplements was the highest in vitamin C (34.7\%), followed by riboflavin (24.4\%), and thiamin (23.9\%), and these values were similar with cancer-free individuals. In French cancer survivors who consumed dietary supplements, the contribution of supplements to total vitamin $C$, riboflavin, and thiamine intakes were $15.6 \%, 9.5 \%$, and $14.3 \%$, respectively [3]. Although the contribution of dietary supplements was high for vitamins D (78.9\%), B6 (44.4\%), and E (35.4\%) in that previous study [3], we could not identify the nutritional contribution of dietary supplements for those nutrients because of the limited nutrient database in our study. However, dietary supplement users tended to have higher blood vitamin D levels, suggesting the significant nutritional contribution of dietary supplements to total vitamin D intakes.

We found that the proportions of participants whose thiamin and niacin intakes below EARs were higher among cancer survivors than cancer-free individuals. The proportions of participants whose calcium intake below EARs were $61.2 \%$ for cancer survivors and $63.0 \%$ for cancer-free individuals, which was the highest rate among nutrients that we examined. The proportions of participants with nutrient intake above ULs were relatively small even after the addition of nutrient intakes from dietary supplements in both cancer survivors $(<4 \%)$ and cancer-free individuals $(<3 \%)$. A previous US study found that approximately $80 \%$ of 753 cancer survivors consumed nutrient amounts below the EARs from foods for vitamin $\mathrm{E}$ and magnesium, and among 559 supplement users, these values were substantially decreased after the consideration of nutrient intakes from supplements: vitamin $\mathrm{E}$ ( $81 \%$ to $12 \%)$ and magnesium $(77 \%$ to $40 \%)$, but proportions of cancer survivors with consumption above ULs were less than 10\% [10].

We examined the factors associated with dietary supplement use among cancer survivors. We found that the proportion of dietary supplement use varied according to the cancer site, and breast cancer had a relatively higher prevalence compared to other cancer sites. These findings were consistent with the results of a systematic review [5]. Several studies have found that, among cancer survivors, high education levels and female sex are associated with the use of dietary supplements $[3,5,10,32]$. We found that education was also associated with the use of dietary supplements among female cancer survivors. Our study found that female cancer survivors who used dietary supplements consumed lower quantities of vegetables compared to non-users. There is evidence that a high consumption of fruits and vegetables is a predictor of the initiation and continuation of vitamin/mineral supplement use [2]. The finding that vegetable intake among supplement users was lower than in non-users warrants further research. Among male cancer survivors, dietary supplement use was associated with living in an urban area, a reduced calorie intake and, no consumption of alcohol. Korean male cancer survivors who use dietary supplements, after the diagnosis of cancer, may have healthier lifestyles compared to non-users of dietary supplements. Further studies with larger numbers of cancer survivors are necessary to identify factors associated with supplement use.

This investigation is the first study, to our knowledge, to estimate the contribution of nutrients from dietary supplements to the total nutrient intake among cancer survivors in a nationwide sample of the population in Korea. Our findings are derived from a representative sample of the community; therefore, the proportion and types of supplements used the quantities of nutrients consumed from dietary supplements, and the characteristics of dietary supplement users may be representative of Korean cancer survivors.

However, our study has several limitations. The number of cancer survivors was small. Therefore, we could not identify the characteristics of supplement users according to the cancer sites, and we could not estimate the nutritional contribution of dietary supplements among specific dietary supplement users. Detailed clinical information, including adjuvant therapy status and cancer stage, was not available. We cannot exclude the possibility that residual and unknown confounding factors may be present. There could be measurement errors from dietary and supplemental assessments, including nutrient intakes from 24-h recalls and dietary supplemental databases. Because we estimated dietary intakes from one-day 24-h dietary recalls, it may not reflect the usual diet of the 
participants. Furthermore, although we assumed that dietary supplement use on the previous day reflects the current usual dietary supplement use, this may not always be true. However, we found similar results in the sensitivity analysis, where we excluded participants who provided discrepant answers on their usual supplement use from the general questionnaire.

\section{Conclusions}

Our results suggest that cancer survivors had high rate of dietary supplement use and higher contribution from dietary supplement to total nutrient intake than cancerfree individuals. Multi-vitamins and minerals were most commonly used, followed by vitamin $\mathrm{C}$, omega-3/fish oil, red ginseng, and calcium among cancer survivors. We observed a higher proportion of participants whose thiamin and niacin intakes below the estimated average requirements among cancer survivors compared to cancerfree individuals. Among cancer survivors, the use of dietary supplements was associated with education levels, physical activity, vegetable intake, calorie intake, living area, and alcohol drinking status. Further studies on the use of dietary supplements among cancer survivors covering a wide range of dietary compounds are warranted. Moreover, prospective and clinical studies are necessary to clarify the association between the use of dietary supplements and cancer prognosis.

\section{Additional files}

Additional file 1: Table S1. Dietary supplement use of study population and according to cancer sites of cancer survivors ${ }^{a}$ without using sampling weight (DOCX $19 \mathrm{~kb}$ )

Additional file 2: Table S2. The proportions of above tolerable upper intake level (UL) among cancer survivors and cancer-free individuals (DOCX $16 \mathrm{~kb})$

\section{Abbreviations \\ 25(OH) D3: 25-hydroxyvitamin D3; 95\% Cls: 95\% confidence intervals; BMI: body mass index; DBBR: databank and biorepository; EARs: Estimated Average Requirements; FFQ: food frequency questionnaires; HDL: high-density lipoprotein; IRB: Institutional Review Board; KCDC: Korea Centers for Disease Control and Prevention; KDRIs: Dietary Reference Intakes for Koreans; KHIDI: Korea Health Industry Development Institute; KMFDS: Korean Ministry of Food and Drug Safety; KNHANES: Korea National Health and Nutrition Examination Survey; KNRLSI: Korean National Rural Living Science Institute; LS-means: Least-squares means; MEC: mobile examination center; NHIS: National Health Interview Surveys; OPMSS: Olestra Post-Marketing Surveillance Study; ORs: odds ratios; SE: standard error; ULs: Tolerable Upper Intake Levels; VITAL: Vitamins and Lifestyle; WHEL: Women's Healthy Eating and Living}

\section{Acknowledgements}

Not applicable.

\section{Funding}

This research was supported by the Basic Science Research Program through the National Research Foundation of Korea (NRF) funded by the Ministry of Science, ICT \& Future Planning (NRF-2014R1A2A2A01007794). The sponsor of the study had no role in study design, data collection, data analysis, data interpretation, or writing of the report. The corresponding author had full access to all the data in the study and had final responsibility for the decision to submit for publication.

\section{Availability of data and materials}

The data are available online at KNHANES website (https://knhanes.cdc.go.kr/ knhanes).

\section{Authors' contributions}

SS contributed to the quality control of data and algorithms, analysis and interpretation of data, and manuscript drafting. JY, YJE, and MK contributed to the quality control of data and algorithms and interpretation of the data TH contributed to the establishment of folate database and the interpretation of the data. YJS contributed to the conception and design, interpretation of the data. JEL contributed to the conception and design, interpretation of the data, manuscript drafting. All authors contributed in data acquisition, revising the manuscript, and final approval of the manuscript.

\section{Ethics approval and consent to participate}

This study was approved by KCDC Institutional Review Board (IRB) Ethics Committee (2010-02CON-21-C, 2011-02CON-06-C) [20]. Informed consent was obtained from all study participants.

\section{Consent for publication}

Not applicable.

\section{Competing interests}

The authors declare that they have no competing interests.

\section{Publisher's Note}

Springer Nature remains neutral with regard to jurisdictional claims in published maps and institutional affiliations.

\section{Author details}

'Department of Food and Nutrition, Seoul National University, Gwanak-ro 1, Gwanak-gu, Seoul 08826, Republic of Korea. ${ }^{2}$ Department of Food and Nutrition, Chungbuk National University, Chungdae-ro 1, Seowon-Gu, Cheongju, Chungbuk 28644, Republic of Korea. ${ }^{3}$ Major of Food and Nutrition, School of Human Ecology, The Catholic University of Korea, 43 Jibong-ro, Wonmi-gu, Bucheon-si, Gyeonggi-do 14662, Republic of Korea.

Received: 1 July 2016 Accepted: 8 December 2017

Published online: 28 December 2017

\section{References}

1. Patterson RE, Neuhouser ML, Hedderson MM, Schwartz SM, Standish L, Bowen DJ. Changes in diet, physical activity, and supplement use among adults diagnosed with cancer. J Am Diet Assoc. 2003;103(3):323-8.

2. Greenlee H, Kwan ML, Ergas IJ, Strizich G, Roh JM, Wilson AT, Lee M, Sherman KJ, Ambrosone CB, Hershman DL, et al. Changes in vitamin and mineral supplement use after breast cancer diagnosis in the pathways study: a prospective cohort study. BMC Cancer. 2014;14:382.

3. Pouchieu C, Fassier P, Druesne-Pecollo N, Zelek L, Bachmann P, Touillaud M, Bairati I, Hercberg S, Galan P, Cohen P, et al. Dietary supplement use among cancer survivors of the NutriNet-Sante cohort study. Br J Nutr. 2015;113(8):1319-29.

4. Bours MJ, Beijer S, Winkels RM, van Duijnhoven FJ, Mols F, Breedveld-Peters JJ, Kampman E, Weijenberg MP, Van de poll-Franse LV. dietary changes and dietary supplement use, and underlying motives for these habits reported by colorectal cancer survivors of the patient reported outcomes following initial treatment and long-term evaluation of survivorship (PROFILES) registry. Br J Nutr. 2015:114(2):286-96.

5. Velicer CM, Ulrich CM. Vitamin and mineral supplement use among US adults after cancer diagnosis: a systematic review. J Clin Oncol. 2008;26(4):665-73.

6. Hardy ML: Dietary supplement use in cancer care: help or harm. Hematol Oncol Clin North Am 2008, 22(4):581-617, vii.

7. Rock CL, Doyle C, Demark-Wahnefried W, Meyerhardt J, Courneya KS, Schwartz AL, Bandera EV, Hamilton KK, Grant B, McCullough M, et al. Nutrition and physical activity guidelines for cancer survivors. CA Cancer J Clin. 2012;62(4):243-74. 
8. Harvie M. Nutritional supplements and cancer: potential benefits and proven harms. American Society of Clinical Oncology educational book / ASCO American Society of Clinical Oncology Meeting. 2014:e478-86.

9. Yasueda A, Urushima H, Ito T. Efficacy and interaction of antioxidant supplements as adjuvant therapy in cancer treatment: a systematic review. Integrative Cancer Therapies. 2015;15(1):17-39.

10. Miller P, Demark-Wahnefried W, Snyder DC, Sloane R, Morey MC, Cohen H, Kranz S, Mitchell DC, Hartman TJ. Dietary supplement use among elderly, long-term cancer survivors. Journal of cancer survivorship : research and practice. 2008;2(3):138-48.

11. Rock CL, Newman V, Flatt SW, Faerber S, Wright FA, Pierce JP. Nutrient intakes from foods and dietary supplements in women at risk for breast cancer recurrence. Nutr Cancer. 1997;29(2):133-9.

12. Lim D, Ha M, Song I. Trends in the leading causes of death in Korea, 1983-2012. J Korean Med Sci. 2014;29(12):1597-603.

13. Jung KW, Won YJ, CM O, Kong HJ, Lee DH, Lee KH. Community of Population-Based Regional Cancer R: cancer statistics in Korea: incidence, mortality, survival, and prevalence in 2014. Cancer research and treatment : official journal of Korean Cancer Association. 2017;49(2):292-305.

14. Ministry of Health and Welfare / Korea Centers for Disease Control and Prevention: Korea Health Statistics 2015 : Korea National Health and Nutrition Examination Survey (KNHANES VI-3). In.; 2016.

15. Kim IK. The use of the functional foods in elderly patients with digestive system cancers. J Korean Gerontol Nurs. 2010;12(1):51-61.

16. Kang HP, Lee H, TG O, Lee KJ, Park SJ, Chung MJ, Kim SU, Lee H, Park JC, Hong SP, et al. The use of health functional foods in gastrointestinal cancer patients. Clin Nutr Res. 2013;2(1):19-25.

17. Kang E, Yang EJ, Kim SM, Chung IY, Han SA, DH K, Nam SJ, Yang JH, Kim SW. Complementary and alternative medicine use and assessment of quality of life in Korean breast cancer patients: a descriptive study. Supportive care in cancer : official journal of the Multinational Association of Supportive Care in Cancer. 2012;20(3):461-73.

18. Hwang JH, Kim W-Y, Ahmed M, Choi S, Kim J, Han DW. the use of complementary and alternative medicine by Korean breast cancer women: is it associated with severity of symptoms? Evid Based Complement Alternat Med. 2015;2015

19. Yun SH, Kim HJ, Oh K. Development of dietary supplement database and nutrient intakes from dietary supplements. Public Health Weekly Report. 2015;8(10):210-6.

20. Korea Centers for Disease Control and Prevention: The Fifth Korea National Health and Nutrition Examination Survey (KNHANES V). In. Seoul, Korea: Korea Centers for Disease Control and Prevention, Ministry of Health and Welfare; 2015.

21. Kweon S, Kim Y, Jang MJ, Kim Y, Kim K, Choi S, Chun C, Khang YH, Oh K. data resource profile: the Korea National Health and nutrition examination survey (KNHANES). Int J Epidemiol. 2014;43(1):69-77.

22. Rural Development Administration: Food composition table, 7th edn. Suwon: Korea National Rural Resources Development Institute; 2006.

23. Kim JH, Lee $E$, Hyun T. Dietary folate intake and food sources of children and adolescents in Chungcheong area - using nutrient database revised by measured folate in selected foods. Journal of Nutr Health. 2015;48(1):94-104.

24. Yon M, Hyun T. Additional data for the folate database for foods common in Korea. Korean J Nutr. 2005;38(7):586-604.

25. The Korean Nutrition Society: Dietary reference intakes for Koreans. In.: the Korean nutrition Society; 2010.

26. Miller MF, Bellizzi KM, Sufian M, Ambs AH, Goldstein MS, Ballard-Barbash R. Dietary supplement use in individuals living with cancer and other chronic conditions: a population-based study. J Am Diet Assoc. 2008;108(3):483-94.

27. McDavid K, Breslow RA, Radimer K. Vitamin/mineral supplementation among cancer survivors: 1987 and 1992 National Health Interview Surveys. Nutr Cancer. 2001;41(1-2):29-32.

28. Greenlee $H$, White $E$, Patterson RE, Kristal AR. Supplement use among cancer survivors in the vitamins and lifestyle (VITAL) study cohort. J Altern Complem Med. 2004;10(4):660-6.

29. LuC L, Baumgart C, Weiss E, Georger L, Ambrosone CB, Zirpoli G, McCann SE. Dietary supplement use among participants of a databank and biorepository at a comprehensive cancer centre. Public Health Nutr. 2014:1-11.

30. Foote JA, Murphy SP, Wilkens LR, Hankin JH, Henderson BE, Kolonel LN. Factors associated with dietary supplement use among healthy adults of five EthnicitiesThe multiethnic cohort study. Am J Epidemiol. 2003;157(10):888-97.
31. Kantor ED, Rehm CD, Du M, White E, Giovannucci EL. Trends in dietary supplement use among us adults from 1999-2012. JAMA. 2016;316(14):1464-74.

32. Miller PE, Vasey JJ, Short PF, Hartman TJ. Dietary supplement use in adult cancer survivors. Oncol Nurs Forum. 2009;36(1):61-8.

33. Ferrucci LM, McCorkle R, Smith T, Stein KD, Cartmel B. Factors related to the use of dietary supplements by cancer survivors. J Altern Complement Med. 2009;15(6):673-80.

34. Rock CL, Newman VA, Neuhouser ML, Major J, Barnett MJ. Antioxidant supplement use in cancer survivors and the general population. J Nutr. 2004;134(11):3194s-5s.

\section{Submit your next manuscript to BioMed Central and we will help you at every step:}

- We accept pre-submission inquiries

- Our selector tool helps you to find the most relevant journal

- We provide round the clock customer support

- Convenient online submission

- Thorough peer review

- Inclusion in PubMed and all major indexing services

- Maximum visibility for your research

Submit your manuscript at www.biomedcentral.com/submit
) Biomed Central 